\title{
Variability and trend of diurnal temperature range in China and their relationship to total cloud cover and sunshine duration
}

\author{
X. Xia \\ LAGEO, Institute of Atmospheric Physics, Chinese Academy of Sciences, Beijing, 100029, China \\ Correspondence to: X. Xia (xxa@mail.iap.ac.cn) \\ Received: 2 November 2012 - Revised: 26 March 2013 - Accepted: 10 April 2013 - Published: 3 May 2013
}

\begin{abstract}
This study aims to investigate the effect of total cloud cover (TCC) and sunshine duration (SSD) in the variation of diurnal temperature range (DTR) in China during 1954-2009. As expected, the inter-annual variation of DTR was mainly determined by TCC. Analysis of trends of 30year moving windows of DTR and TCC time series showed that TCC changes could account for that of DTR in some cases. However, TCC decreased during 1954-2009, which did not support DTR reduction across China. DTRs under sky conditions such as clear, cloudy and overcast showed nearly the same decreasing rate that completely accounted for the overall DTR reduction. Nevertheless, correlation between SSD and DTR was weak and not significant under clear sky conditions in which aerosol direct radiative effect should be dominant. Furthermore, 30-60\% of DTR reduction was associated with DTR decrease under overcast conditions in south China. This implies that aerosol direct radiative effect appears not to be one of the main factors determining long-term changes in DTR in China.
\end{abstract}

Keywords. Atmospheric composition and structure (Cloud physics and chemistry)

\section{Introduction}

The annual average global temperature increased $0.74 \pm$ $0.18^{\circ} \mathrm{C}$ from 1906 to 2005 (IPCC, 2007). However, the diurnal temperature range (DTR) was observed to decrease since the 1950s over most global land areas due to a lower rate of warming in maximum temperature than in minimum temperatures (Karl et al., 1993; Zhou et al., 2004). Cloud and precipitation are widely suggested to have played an important role in the worldwide DTR reduction due to their significant effects on the surface energy and hydrological balance (Karl et al., 1993; Dai et al., 1997, 1999). Clouds cool the earth's surface by reflecting sunlight during daytime and warm the surface by emitting longwave radiation, and thereby exert a damping effect on DTR. Thick clouds with low bases are most effective in reflecting sunlight and radiate more longwave radiation back to earth. Therefore thick clouds are very effective in damping DTR, while middle and high clouds have only moderate damping effects on DTR (Dai et al., 1997, 1999). Coincident increases in cloud cover found in many locations have been cited as a plausible cause for the DTR reduction (Karl et al., 1993). Precipitation also impacts DTR to some extent (Dai et al., 1997; Zhou et al., 2004). Data analysis showed that the long-term DTR trend exhibited a significant correlation with precipitation amount. Stronger DTR decreasing trends were observed over drier regions (Zhou et al., 2010). Up to $80 \%$ of the DTR variance was explained by the cloud and precipitation records (Dai et al., 1999). DTR is also impacted by changes of surface properties, greenhouse gases and aerosols.

Model simulations showed that none of the known globally distributed climate forcing could account for the observed DTR changes. A larger global warming mechanism coupled with a forcing concentrated more in the continental regions could yield the observed DTR reduction (Hansen and Sato, 1995). Analysis of data from model simulations during the 20th century $(20 \mathrm{C} 3 \mathrm{M})$ showed that these models reproduced the overall 20th century warming over global land surfaces well but underestimated the decadal variations in the DTR and surface solar radiation (SSR). This lack in decadal variability in the model-calculated DTR and SSR could have various origins (Wild, 2009a). An obvious origin could be a missing increasing trend in the modeled cloud characteristics, such as cloud cover and cloud albedo, which could likely result from a poor representation of complex aerosol-cloud 


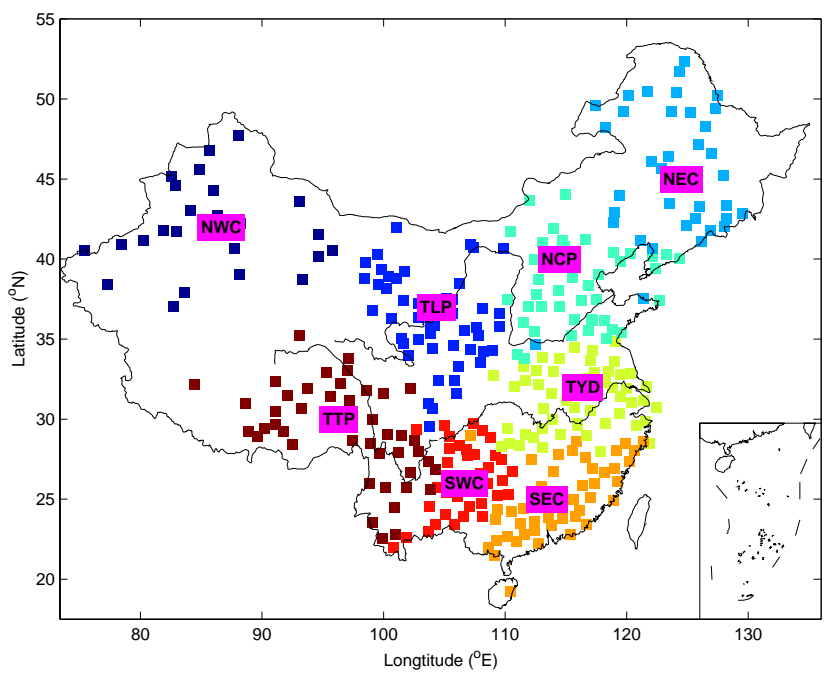

Fig. 1. Spatial distribution of 332 surface observation stations in China used in the analysis. Eight regions are classified using a factor analysis with varimax rotation including (1) Northwest China (NWC), (2) the Loess Plateau (TLP), (3) Northeast China (NEC), (4) the North China Plain (NCP), (5) the Yangtze Delta region (TYD), (6) Southeast China (SEC), (7) Southwest China (SWC), and (8) the Tibetan Plateau (TTP).

interactions in the models or our poor understanding of cloud feedbacks to global warming. Furthermore, natural variability could also potentially induce decadal-scale variations in cloud properties, SSR and DTR, which were also likely not reproduced by the models to their full extent (Wild, 2009b).

The warming rate of annual mean surface air temperature in China for the last half century was estimated to be $0.22^{\circ} \mathrm{C} /$ decade, which was more rapid than the average values of the world and the Northern Hemisphere (Guo et al., 2005). The DTR decreased by $0.25^{\circ} \mathrm{C} /$ decade during 1960 1990 as a result of increasing minimum temperature and slightly decreasing maximum temperature; however, DTR in the 1990s was observed to level off (Liu et al., 2004). Correlation analysis showed that changes of cloud cover and precipitation could not account for the decreased DTR (Liu et al., 2004). This led to an argument that it was likely attributable to the global dimming induced by increased aerosols (Liu et al., 2004; Che et al., 2005; Liang and Xia, 2005).

This study is intended to answer the following questions: (1) What is the spatio-temporal variability of DTR during 1954-2009 under sky conditions such as clear, cloudy and overcast? (2) What percent of DTR trends can be accounted for by changes in total cloud cover (TCC)? (3) What is the relationship between DTR and sunshine duration (SSD) and its implication for aerosol direct radiative effect? This kind of analysis may provide better understanding how and why DTR in China varied over the past 50 years.

\section{Data and methodology}

\subsection{Data}

The measurements of maximum and minimum temperature, cloud and sunshine duration were managed by the China Meteorological Administration (CMA). Daily surface observations of these quantities at more than 700 meteorological sites are archived and available from the Climate Data Center (CDC) of the CMA. Quality control checks were performed by CDC/CMA. Ground-based observations of TCC in units of percent of sky cover are based on subjective estimates of cloud amount at 6-h intervals, 00:00, 06:00, 14:00, 22:00 UTC, by experienced individuals. SSD, the time during which the direct solar irradiance exceeds $120 \mathrm{~W} \mathrm{~m}^{-2}$ as defined by the World Meteorology Organization, is observed using the sunshine recorder. The DTR was calculated as the difference between the maximum and minimum temperature.

\subsection{Testing the homogeneity of the DTR series}

Climate observations are sometimes impacted by nonclimatic factors such as changes in station location, exposure of the observational site, instrumentation type, or measurement procedure, thereby leading to abrupt or gradual changes in data records. The RHtestV3 software package in which the penalized maximal $t$ test and the penalized maximal $F$ test were embedded was used here to detect multiple shifts in a DTR series that displayed first-order autoregressive errors (Wang, 2008). The RHtestV3 was applied to each site's data to detect shifts (if present) without using a reference series. The DTR data proved to be homogeneous at 332 out of 666 sites having data of more than 30 years. There is no metadata available for determining whether the shift detected by the RHtestV3 is real. Therefore, only homogeneous time series at 332 sites (Fig. 1) were used to minimize effects of data inhomogenity on the analysis.

\subsection{Regionalization}

A network of stations with sufficient density to determine mean climatic values is suitable for detection of climatic change. Therefore, a factor analysis with varimax rotation is applied to group the 332 sites into 8 subregions with similar DTR variability (Sanchez-Lorenzo et al., 2007). Figure 1 presents the identified subregions: (1) Northwest China (NWC), (2) the Loess Plateau (TLP), (3) Northeast China (NEC), (4) the North China Plain (NCP), (5) the Yangtze Delta region (TYD), (6) Southeast China (SEC), (7) Southwest China (SWC), and (8) the Tibetan Plateau (TTP). Prior to analysis, the data from individual sites were converted to departures from normal, with normal approximated by period means during 1971-2000. This allowed for the calculation of seasonal averages from adjacent months and the calculation of regional averages from individual sites, thus increasing the number of cases and the reliability of the 
statistically derived relationships. The monthly anomalies have been averaged by season (winter is defined as December, January, and February, spring as March, April, and May, summer as June, July, and August, and autumn as September, October, and November), and an annual average has been obtained from the average of the four seasons.

\subsection{Statistical method for trend analysis}

Detection of long-term linear trends in the geophysical data needs to account for the magnitude of variability and autocorrelation of the noise in the data. Since autocorrelation is often evident in the meteorological data, a trend analysis formulation is used here to specifically account for this autocorrelation. The method is firstly introduced by Weatherhead et al. (1998) to analyze monthly anomaly, and extended by Hinkelman et al. (2009) for annual time series. A brief introduction is presented for the completeness. The annual anomaly is assumed to fit a trend model of the form

$Y_{t}=\mu+\omega X_{t}+N_{t}$,

where $Y$ is the annual DTR, $\mu$ is a constant term, $\omega$ is the magnitude of the trend per year, $t$ is the index of annual samples, $X_{t}=t$ represents the linear trend function, and $N_{\mathrm{t}}$ is the noise or the part of the time series not explained by the linear trend. The noise is assumed to be $\operatorname{AR}(1)$, so that $N_{t}=\varphi N_{t-1}+\varepsilon_{t}$, where $|\varphi|<1$ and the $\varepsilon_{t}$ values are independent random variables with mean zero and variance $\sigma_{\varepsilon}^{2}$. The trend, $\omega$, is then estimated using a standard least squares method, and its standard deviation is calculated according to the following equation: $\sigma_{\mathrm{w}} \approx \sigma_{N} \sqrt{\frac{12}{n^{3}}} \sqrt{\frac{1+\varphi}{1-\varphi}}$, where $n$ is the number of years of data in the series and $\sigma_{N}^{2}=\sigma_{\varepsilon}^{2} /\left(1-\varphi^{2}\right)$. As implied by these equations, it is obviously more difficult to detect a trend with confidence when the noise on the signal is large or when the signal is highly autocorrelated.

\subsection{Quantitatively estimated contribution of TCC to the DTR trend}

The DTR is generally greater on clear days than on cloudy or overcast days as a result of cloud damping effect on the DTR. Therefore, the more clear days, the larger the average DTR is and vice versa. A simple yet effective method was used here to evaluate how much the DTR trend is attributable to changes in the frequency of different sky conditions and how much is contributed by changes in the average DTR associated with each sky condition. The daily data at each station were grouped into 5 different sky conditions according to TCC values, i.e., TCC within 0-20, 20-40, 40-60, 6080, 80-100 percents (Xia, 2010a). DTR data are stratified by these cloud cover categories to study how and why DTR has changed (Sanchez-Lorenzo and Wild, 2012; SanchezLorenzo et al., 2012). The relationship between the overall
DTR trend can be derived as follows.

$365 \times \overline{\mathrm{DTR}}_{y}=\sum_{i=1}^{5} \overline{\mathrm{DTR}_{i y}} \times N D_{i y}$,

where $\overline{\mathrm{DTR}}_{y}$ is the annual DTR average for one year $y$. $\overline{\mathrm{DTR}}_{i y}$ is the annual average DTR for the $i$-th TCC group, and its occurrence is represented by $N D_{i y}$. The first derivative of the equation with regards to time is then calculated to derive the following equation.

$365 \times \omega_{\mathrm{DTR}}=\sum_{i=1}^{5}\left(\omega_{\mathrm{DTR}_{i}} \times \overline{\mathrm{ND}_{i}}+\omega_{\mathrm{NUM}_{i}} \times \overline{\mathrm{DTR}_{i}}\right)$,

where $\omega$ represents the linear trend. Therefore, based on the Eq. (3), we can quantitatively estimate how much of the overall DTR trend $\left(\omega_{\mathrm{DTR}}\right)$ is likely accounted for by change in the occurrence frequency of each sky condition $\left(\omega_{N D_{i}}\right)$ and how much by change in its DTR average $\left(\omega_{\mathrm{DTR}_{i}}\right)$. Percentage of contribution by TCC frequency changes with the overall DTR trend, i.e., $\omega_{\text {DTR }}$ can be derived using following equation: $\sum_{i=1}^{5}\left(\omega_{N D_{i}} \times \overline{\mathrm{DTR}_{i}}\right) /\left(365 \times \omega_{\mathrm{DTR}}\right) \times 100$.

\section{Results}

\subsection{Variability in DTR and TCC and their relationship at different timescales}

Figure 2 shows the time series of seasonal and annual DTR and TCC in the eight sub-regions, together with their fits using the robust locally weighted regression algorithm "Lowess" for a better visualization of the decadal variability. The DTR and TCC trends were calculated by means of least squares linear fitting, and the significance was estimated using the Weatherhead et al. (1998) method. The italic and bold formatting of values represent that the trends are significant at the $99 \%$ level. The outstanding feature was that both DTR and TCC showed a decreasing trend in all regions. Furthermore, the DTR reduction in spring (drier season) and in north China (drier regions) was generally larger than that in summer and in south China. This feature is in agreement with the results of Zhou et al. (2010) who pointed out that the magnitude of the decreasing DTR trend decreased with increasing precipitation amount and cloud cover. Figure 3 shows the annual and seasonal China series of TCC and DTR, together with the decadal variability. Annual DTR and TCC decreased by $0.14^{\circ}$ and $0.69 \%$ per decade during 1954-2009, respectively. The maximum and minimum DTR trend $(-0.21$ and $-0.09^{\circ}$ per decade) occurred in winter and summer, respectively. Note that the China DTR has leveled off since the 1990s and even showed a slight increasing trend in spring. This feature was also evident on the regional time series. 


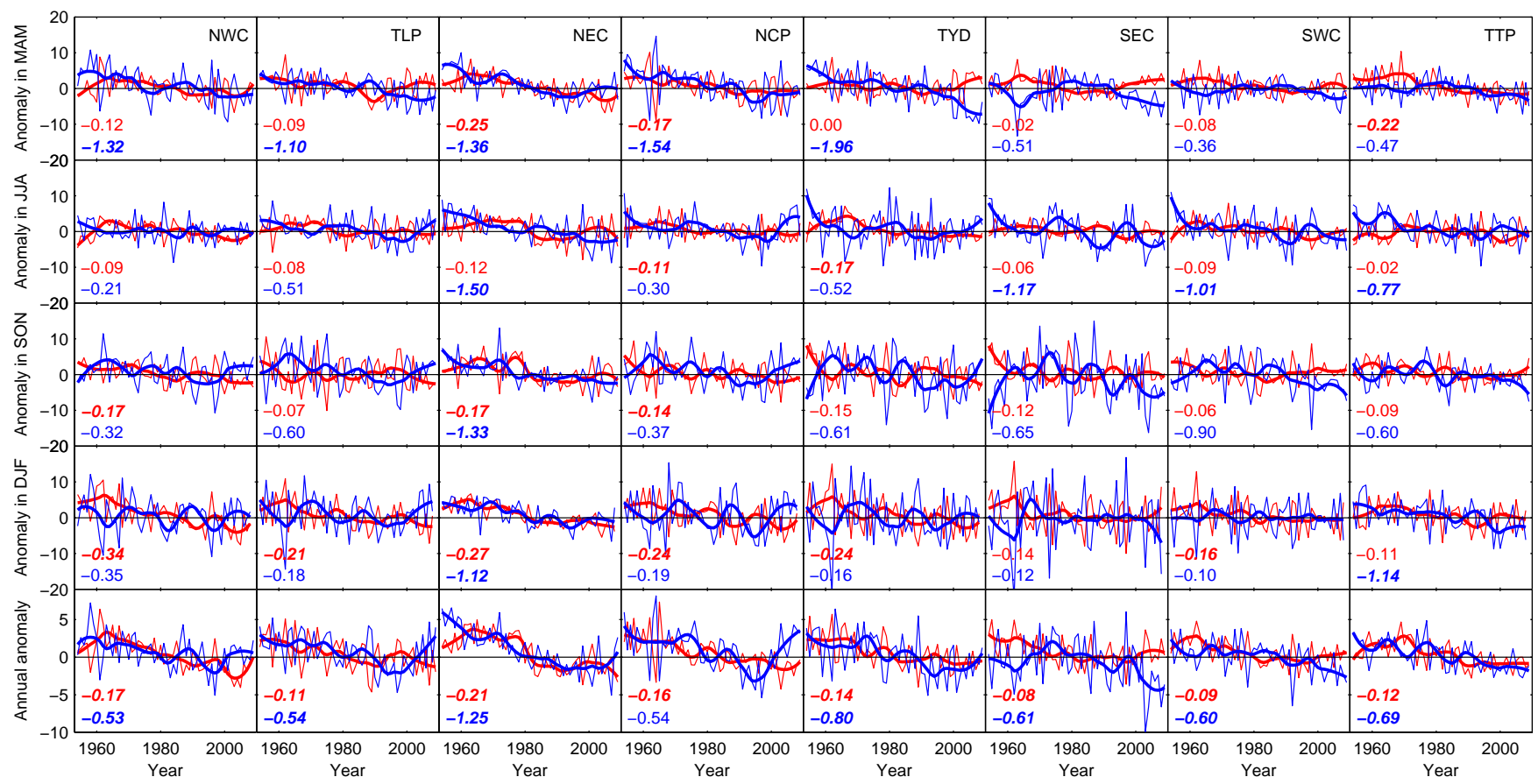

Fig. 2. Time series plots of average regional anomalies of diurnal temperature range (red) and total cloud cover (blue), plotted together with the smoothed series using robust locally weighted regression algorithm "Lowess". The long-term trends of diurnal temperature range and cloud cover are also included in the figure and the bold/italic values represents that the trends are significant at the $99 \%$ level.

Although DTR and TCC showed a decreasing trend during 1954-2009, it was still shown that short-term DTR variability co-varied inversely with that of TCC. In order to distinguish between the decadal and inter-annual agreement of the two measures, we subtracted the raw time series of DTR and TCC by the smoothed series to obtain inter-annual variability and then calculated the correlation separately. Figure 4 presents the correlation coefficients at high (inter-annual) and low (decadal) frequency. The correlation between the interannual variations of DTR and TCC in all regions was significant at the $99 \%$ level, which was likely because they were determined by the variability of synoptic systems (Makowski et al., 2009). Also shown was an inverse co-variation of decadal changes in DTR and TCC in south China (Fig. 4). As a matter of fact, one can see that the regional trends of DTR and their correlations with that of TCC depend on the selected period in Fig. 2. Thus in order to extract as much information as possible from the data, a trend analysis was also applied on running windows for the annual and seasonal series. The trends of DTR and TCC were estimated within temporal windows of 30 years (Sanchez-Lorenzo et al., 2007). Figure 5 presents the scatter plot of trends of both measures based on the annual and seasonal time series of running windows. The dominant feature is that DTR generally shows a downward tendency, which is often associated with a reduction of TCC. However, a few interesting features should also be noted. First, one can still see an increasing DTR tendency in some regions during some periods, for example, during the early period in north China (mostly in spring) as well as during the later period in south China (mostly in spring and fall). Second, the increasing trend of DTR is always associated with a decreasing trend of TCC. Third, DTR reduction during the early period in SWC and SEC and during the later period in the NCP is accompanied by an increase of TCC, indicating TCC still can be used to explain DTR decadal variability during these periods although the overall trends of DTR and TCC during 1954-2009 show the same tendency.

\subsection{Contribution of TCC variations to changes in DTR}

A brief picture of long-term changes in DTR under all sky conditions has been presented; however, a rather fundamental question still needs further analysis. Specifically, we would like to know how much of any DTR changes under all sky conditions is attributable to changes in the frequency of clear skies and/or cloudy skies and how much is contributed by the average DTR. This analysis can help us to understand the potential causes for the DTR changes. By means of a statistical analysis method described above, a quantitative estimate of the proportion of any trend in DTR that is attributable to changes in sky conditions versus changes in its average DTR is shown in Fig. 6. One would expect that long-term reduction of TCC has resulted in an increase of DTR. However, a decreasing trend of DTR was observed. This is completely resulted from DTR reduction under all sky conditions. This 


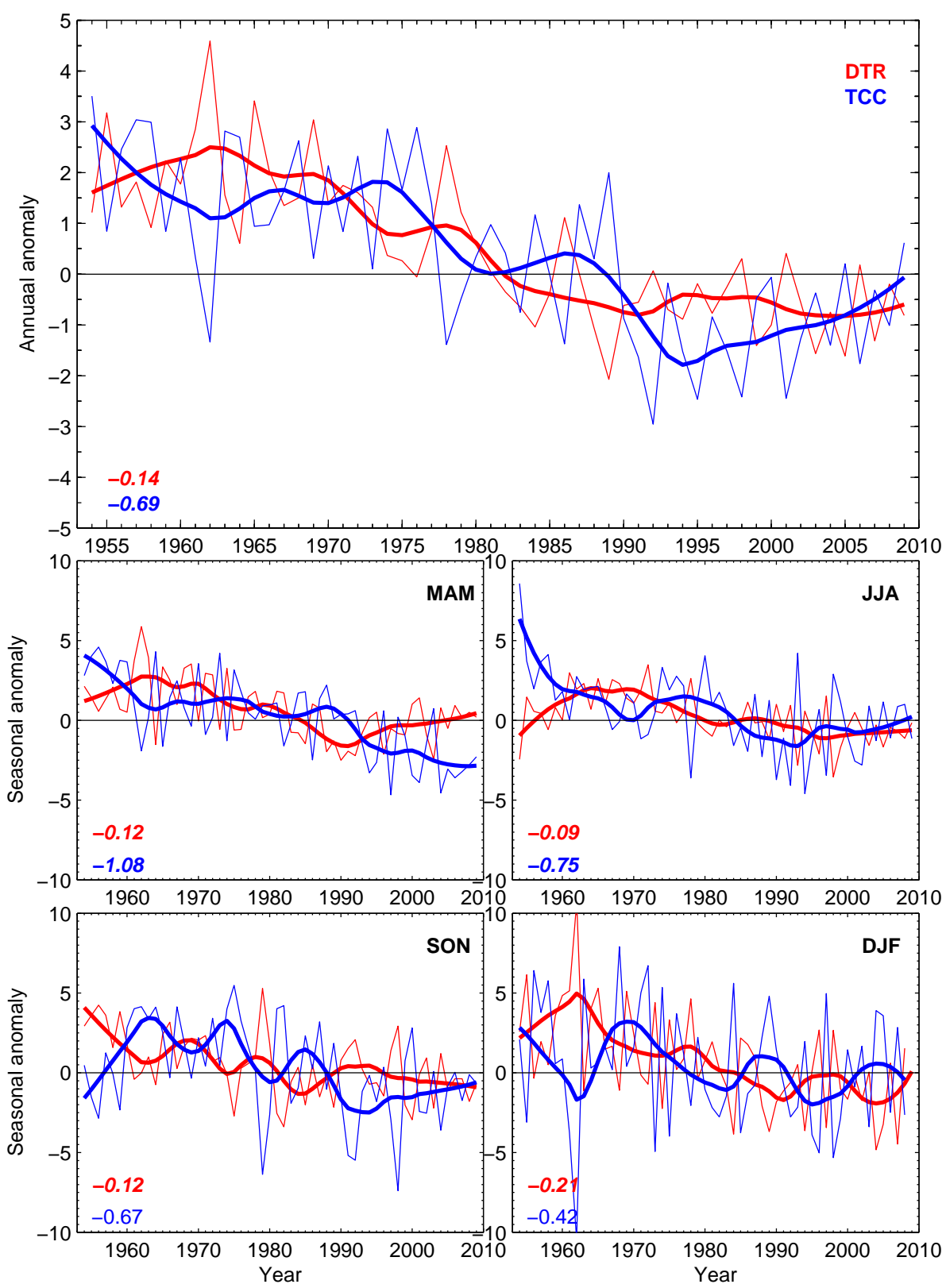

Fig. 3. Same as Fig. 2 but for the average China series.

is clearly shown by Fig. 7 in which long-term trends in average DTRs for each sky condition are presented. The solid dots represent that the trend is significant at the $99 \%$ level. Nearly all average DTRs for each sky condition reduced significantly at the $99 \%$ level except in a few cases that occurred in SWC and SEC. The DTR trends showed little dependence on sky condition. Figure 8 presents contributions of changes in average DTRs for each sky condition to the overall DTR trends. A quite similar contribution was observed in north China and in the TTP; however, in south China the proportion of the DTR reduction attributable to overcast conditions, i.e., cloud cover within $80-100 \%$, ranged from $30 \%$ to $65 \%$, which depended on season. This is mostly because the frequency of overcast conditions is dominant in south China (not shown), although the average DTR for this sky condition shows nearly the same reduction rate as compared with other sky conditions.

\subsection{Correlation between DTR and SSD}

It was suggested that variability of SSR in China was closely related to that of DTR (Liu et al., 2004), although they used SSR data at 85 pyranometer stations. Here, I use SSD measurements, a good proxy for surface irradiance 

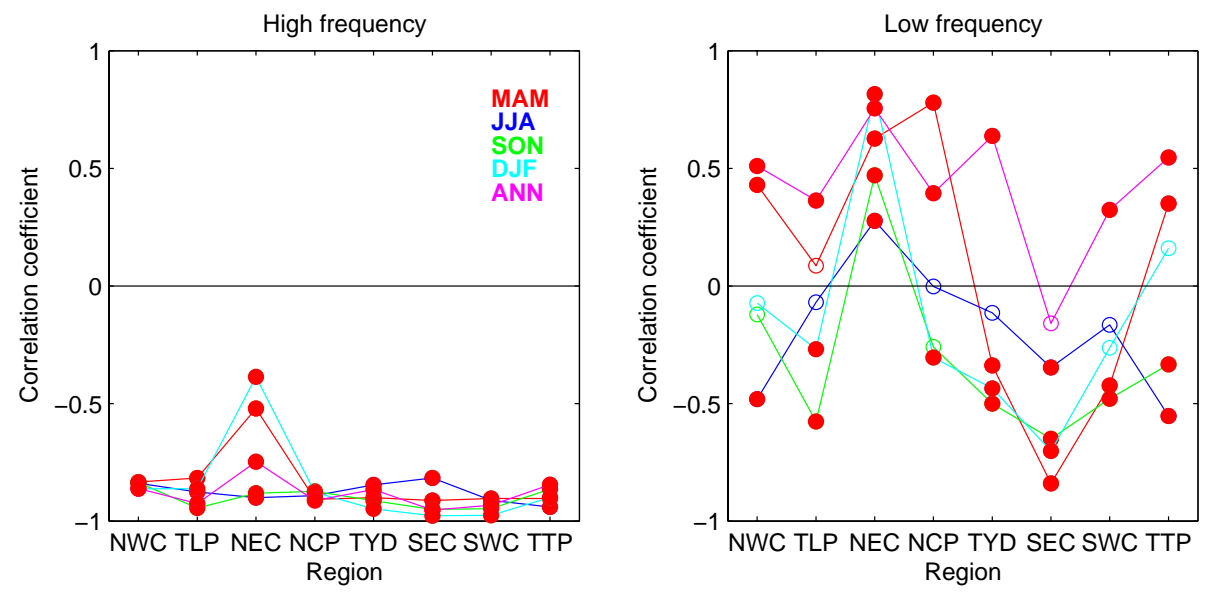

Fig. 4. Correlation coefficients of high (left) and low (right) frequency between diurnal temperature range and total cloud cover.

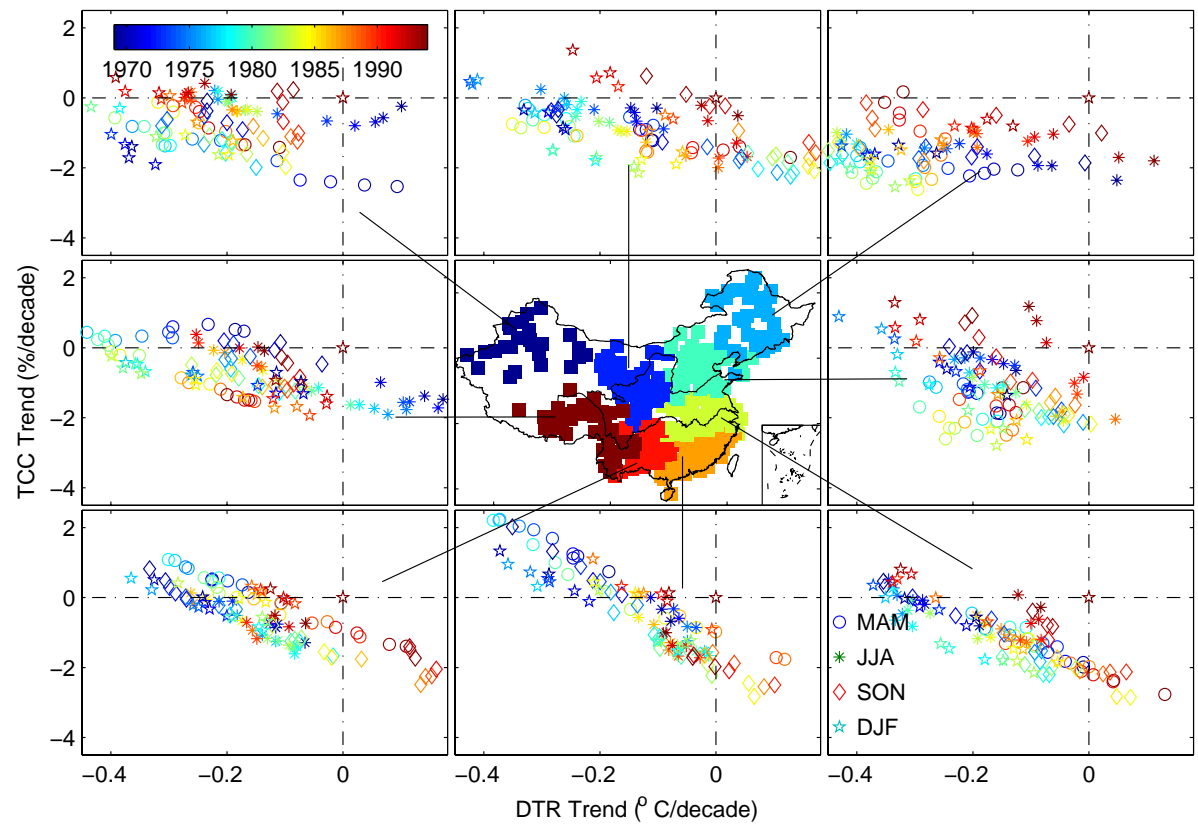

Fig. 5. Scatter-plot of trends of diurnal temperature range and total cloud cover based on 30-year windows of the time series.

(Sanchez-Lorenzo and Wild, 2012; Wang et al., 2012), to study co-variation of SSD and DTR. The advantage of using SSD is that SSD and DTR measurements are available at all stations. Figure 9 shows the correlation coefficients between DTR and SSD variability for each sky condition during 1954-2009. A significant positive correlation was always derived under any sky condition. An interesting feature is that the correlation between DTR and SSD for clear skies (TCC within 0-20\%) was generally less than that for cloudy and overcast skies. This feature was more prominent in south China and in seasons with relatively larger SSD. This implied that variability of SSR under this condition appeared not to play an important role in DTR variability.

\section{Discussion}

Cloud and precipitation is widely used to explain DTR variability (Dai et al., 1997, and references therein); however, DTR and TCC showed the same decreasing trend in China, which implied that factors other than TCC were required to explain DTR variability. SSR showed a positive correlation to DTR in China; therefore, it was used to explain variability of DTR (Liu et al., 2004). Clouds and aerosols are important modulators of SSR. The general picture of the long-term behavior of SSR during 1961-2005 in China is that dimming is accompanied by a reduction in TCC (Xia, 2010b). It was widely suggested that aerosol loading increased due to rapid increase of anthropogenic emissions (Qiu and Yang, 2000; 


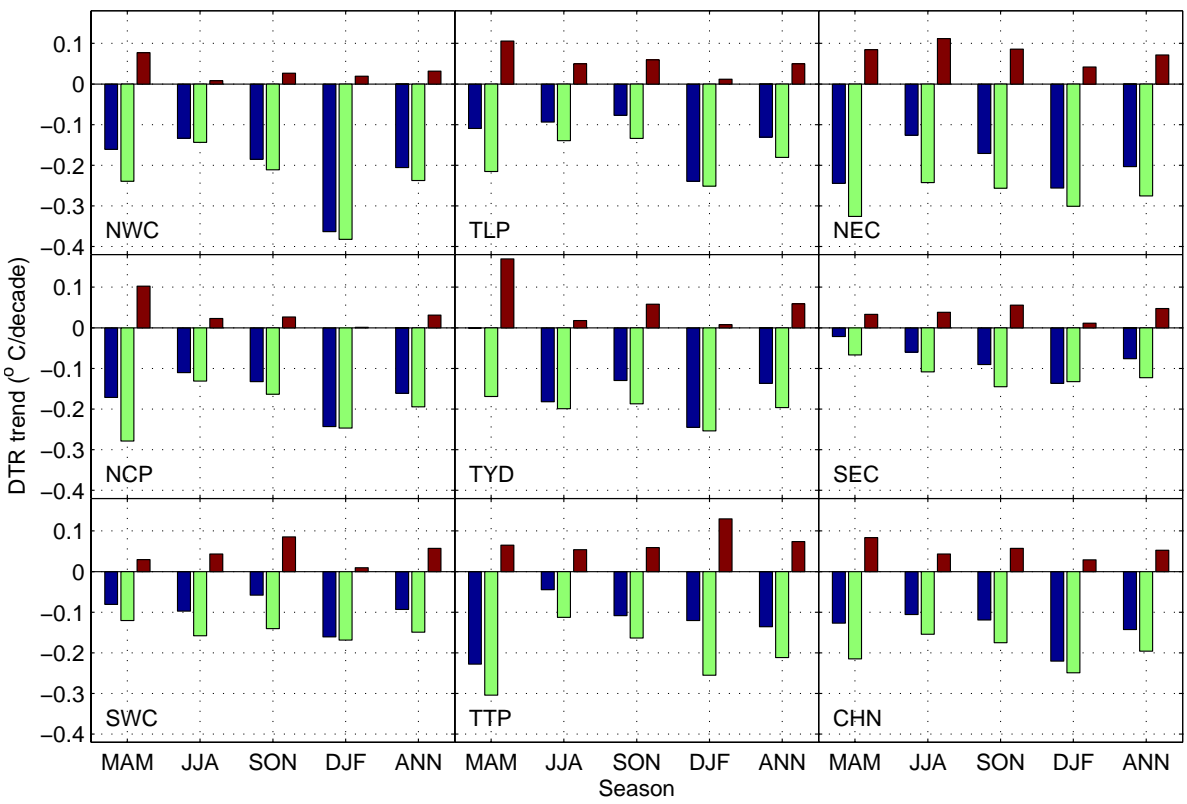

Fig. 6. Long-term trends of diurnal temperature range (blue) and contributions by changes in diurnal temperature range (green) and cloud cover (purple).

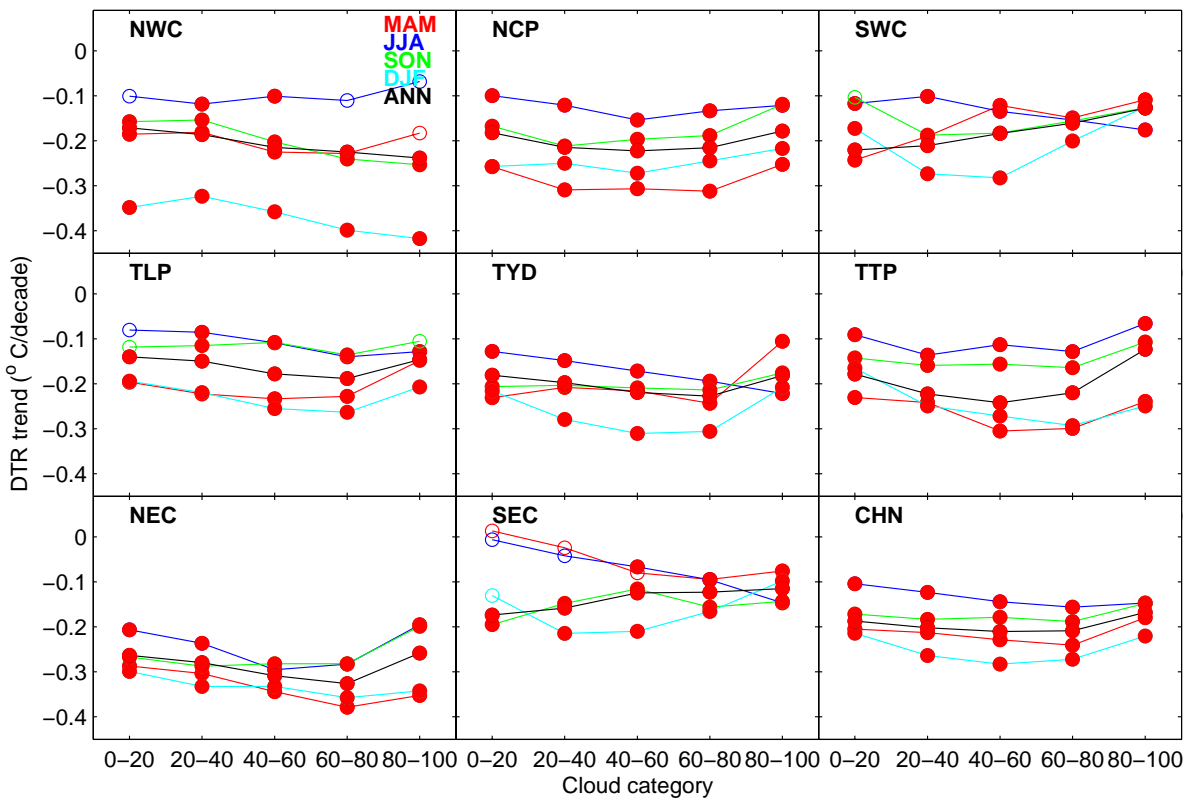

Fig. 7. Long-term trends of diurnal temperature range during 1954-2009 for different sky conditions characterized by total cloud cover. The solid circles represent that the trend is significant at the $99 \%$ level.

Luo et al., 2001; Xia et al., 2007). Increasing aerosol loading might account for dimming, and thereby reduce DTR in China (Liu et al., 2004; Che et al., 2005). However, it should be pointed out that the correlation between the long-term behavior of SSR and TCC is somewhat complex. The regional trends of SSR and their correlation with that of clouds depend on the selected period. Significant dimming during 19601990 is mostly accompanied with TCC reduction. However, one can still see a good inverse co-variability of decadal changes in SSR and TCC since 1990s when a portion of dimming should be related to TCC variability (Xia, 2010b).

SSD is positively correlated to DTR under all skies, but the correlation coefficients under relatively clear skies are generally less than those derived under cloudy skies, indicating that the relationship between DTR and SSD under cloudy skies is more prominent. Aerosol can induce a substantial 


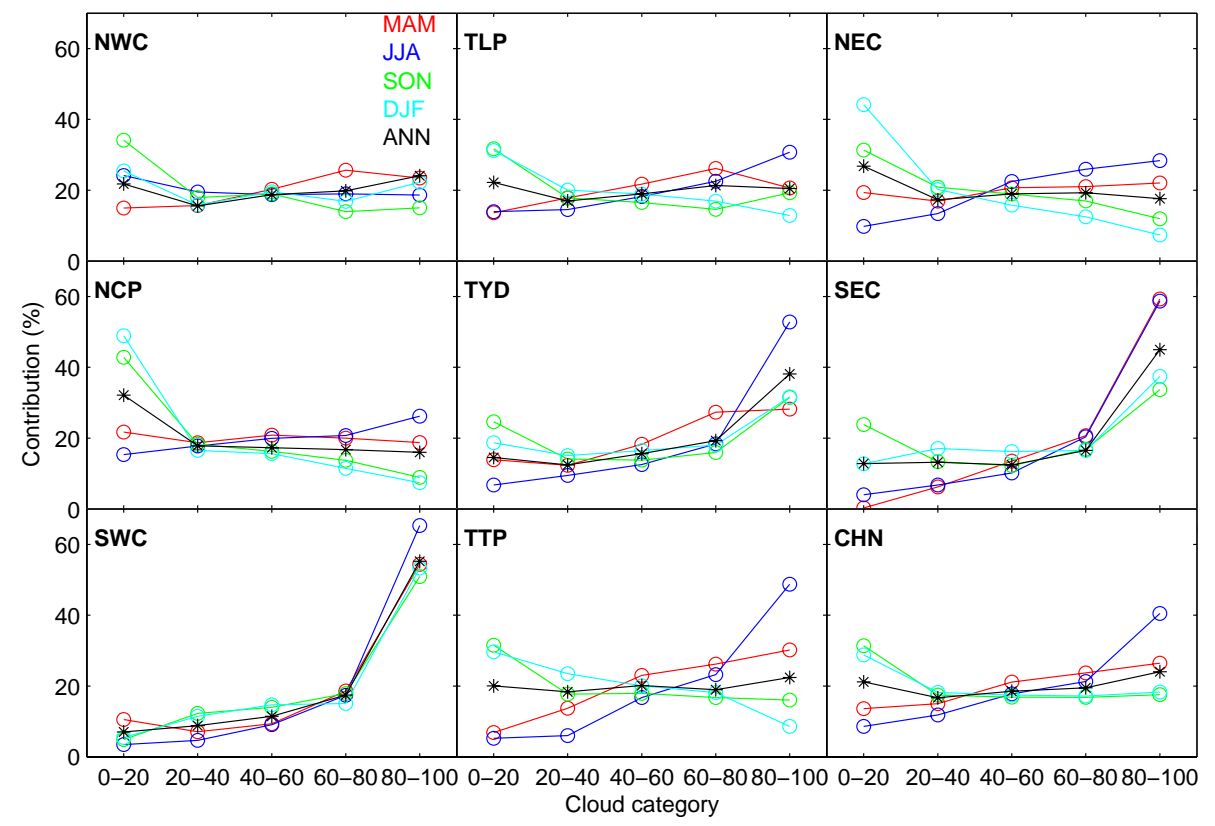

Fig. 8. Contribution of changes in diurnal temperature range for different sky conditions to the overall diurnal temperature range.

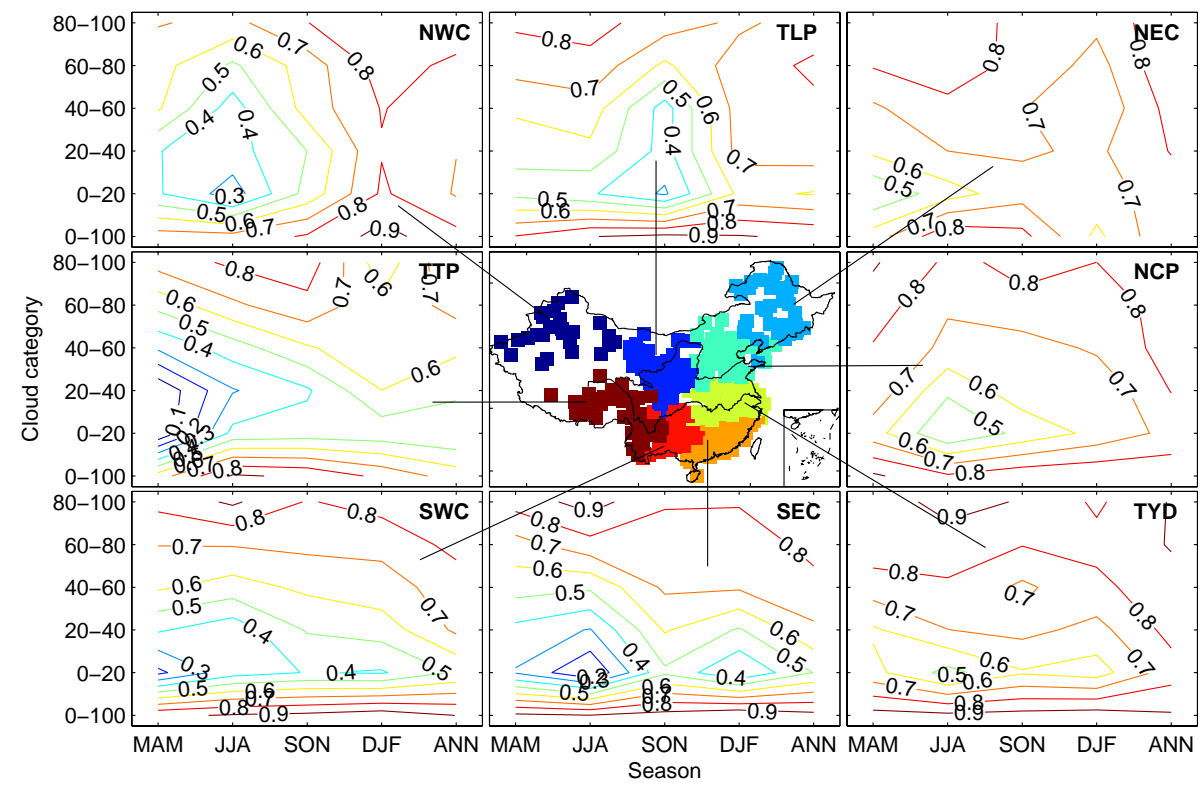

Fig. 9. Correlation coefficients between seasonal and annual series of diurnal temperature range and sunshine duration.

decrease in SSR via its scattering and absorption of sunlight. However, aerosol direct effect on SSR should decrease with TCC because an increase of TCC and thereby TCC scattering leads to less solar radiation available for aerosol scattering and absorption. Given that there is a relatively poor correlation between DTR and SSD and aerosol direct effect on SSR is stronger in clear skies, aerosol direct radiative effect should not play an important role in determining DTR variability in China. In particular, the frequency of overcast conditions (TCC $>80 \%$ ) in south China is close to $60 \%$, under which condition DTR reduction should not maintain close ties with small aerosol direct radiative effect. Aerosols, as cloud condensation nuclei, can impact cloud microphysical and radiative properties via its indirect effects and thereby impact DTR. A regional climate-chemistry-aerosol model simulation showed that changes in downward longwave radiation at the surface were important in understanding DTR changes from aerosols (Huang et al., 2006). Further analysis of whether the observed DTR reduction under cloudy and 
overcast conditions was associated with aerosol indirect effect is required.

\section{Conclusions}

In this study, the long-term variability and trend of a homogeneous DTR series in China and potential causes are addressed during the period 1954-2009. The role TCC played in DTR variability is studied by determining the degree of agreement between the high and low frequency parts. A statistical method is applied to estimate how much DTR change is attributable to TCC frequency change.

There is a very complex spatio-temporal DTR variability. The general picture is that DTR shows a negative tendency during 1954-2009. Annual DTR has decreased by $0.14^{\circ}$ per decade during 1954-2009 for the national average. The relatively larger decreases in DTR occur in arid and semi-arid regions and during dry seasons.

Running trend analysis of 30-year windows of DTR shows an upward tendency for the early spring series in north China and for the later spring and fall series in south China. Those regions showing increasing trends of DTR are associated with downward TCC. Further, TCC can explain DTR variability during the early period in south China. However, longterm TCC reduction during 1954-2009 should have resulted into an increase of DTR that has been completely offset by the nearly same decrease rate of DTR under all sky conditions.

A significant positive correlation between SSD and DTR is derived. The correlation under clear sky conditions is generally less than that for cloudy and overcast skies. This feature is more prominent in regions and seasons with larger $\mathrm{SSD}$, indicating that surface solar radiation variability due to aerosol direct radiative effect under this condition appears not play an important role in DTR variability.

Acknowledgements. The surface observation data of cloud, temperature, and sunshine duration are obtained from the Climate Data Center, Chinese Meteorological Administration. The author appreciates Xiaolan L. Wang at the Climate Research Division, Atmospheric Science and Technology Directorate, Science and Technology Branch, Environment Canada, for providing the RHtestV3 algorithm. The research was supported by the Knowledge Innovation Program of the Chinese Academy of Sciences (KZCX2-YX-QN201) and the National Science Foundation of China (NSFC41175031).

Topical Editor P. Drobinski thanks two anonymous referees for their help in evaluating this paper.

\section{References}

Che, H., Shi, G., Zhang, X., Arimoto, R., Zhao, J., Xu, L., Wang, B., and Chen, Z.: Analysis of 40 years of solar radiation data from China, 1961-2000, Geophys. Res., Lett., 32, L06803, doi:10.1029/2004GL022322, 2005.
Dai, A., Del Genio, A., and Fung, I.: Clouds, precipitation, and temperature range, Nature, 386, 665-666, 1997.

Dai, A., Trenberth, K., and Karl, T.: Effects of clouds, soil moisture, precipitation, and water vapor on diurnal temperature range, J. Climate, 12, 2451-2473, 1999.

Guo, R., Guo, J., Xu, M., Chu, Z., Zhang, L., Zou, X., Li, Q., and Liu, X.: Climate changes of China's mainland over the past half century, ACTA Meteo. Sin., 63, 942-956, 2005.

Hansen, J. and Sato, R.: Long-term change of the diurnal temperature cycle: implications about mechanisms of global climate change, Atmos. Res., 37, 175-209, 1995.

Hinkelman L., Stackhouse, P., Wielicki, B., Zhang, T., and Wilson, S.: Surface insolation trends from satellite and ground measurements: comparisons and challenges, J. Geophys. Res., 114, D00D20, doi:10.1029/2008JD011004, 2009.

Huang, Y., Dickinson, R., Chameides, W.: Impacts of aerosol indirect effect on surface temperature over East Asia, Proc. Natl. Acad. Sci. USA, 103, 4371-4376, 2006.

IPCC, Climate change 2007: the physical science basis, contribution of working group I to the fourth assessment report of the IPCC (ISBN 9780521 88009-1), Cambridge University Press, Cambridge, 2007.

Karl, T., Knight, R., Gallo, K., Peterson, T., Jones, P., Kukla, G., Plummer, N., Razuvayev, V., Lindseay, J., and Charson, R.: A new perspective on recent global warming: asymmetric trends of daily maximum and minimum temperature, B. Am. Meteorol. Soc., 74, 1007-1023, 1993.

Liang, F. and Xia, X. A.: Long-term trends in solar radiation and the associated climatic factors over China for 1961-2000, Ann. Geophys., 23, 2425-2432, doi:10.5194/angeo-23-2425-2005, 2005.

Liu, B., Xu, M., Henderson, M., Qi, Y., and Li, Y.: Taking China's temperature: daily range, warming trends, and regional variations, 1955-2000, J. Climate, 17, 4453-4462, 2004.

Luo, Y., Lu, D., Zhou, X., Li, W., and He, Q.: Characteristics of the spatial distribution and yearly variation of aerosol optical depth over China in last 30 years, J. Geophys. Res., 106, 14501-14513, 2001.

Makowski, K., Jaeger, E. B., Chiacchio, M., Wild, M., Ewen, T., and Ohmura, A.: On the relationship between diurnal temperature range and surface solar radiation in Europe, J. Geophys. Res., 114, D00D07, doi:10.1029/2008JD011104, 2009.

Qiu, J. and Yang, L.: Variation characteristics of atmospheric aerosol optical depths and visibility in North China during 19801994, Atmos. Environ., 34, 603-609, 2000.

Sanchez-Lorenzo, A. and Wild, M.: Decadal variations in estimated surface solar radiation over Switzerland since the late 19th century, Atmos. Chem. Phys., 12, 8635-8644, doi:10.5194/acp-128635-2012, 2012.

Sanchez-Lorenzo, A., Brunetti, M., Calbo, J., and Martin-Vide, J.: Recent spatial and temporal variability and trends of sunshine duration over the Iberian Peninsula from a homogenized data set, J. Geophys. Res., 112, D20115, doi:10.1029/2007JD008677, 2007.

Sanchez-Lorenzo, A., Calbó, J., and Wild, M.: Increasing cloud cover in the 20th century: review and new findings in Spain, Clim. Past, 8, 1199-1212, doi:10.5194/cp-8-1199-2012, 2012.

Wang, X.: Accounting for autocorrelation in detecting mean shifts in climate data series using the penalized maximal $t$ or $F$ test, J. Appl. Meteorol. Climatol., 47, 2423-2444, doi:10.1175/2008JAMC1741.1, 2008. 
Wang, K. C., Dickinson, R. E., Wild, M., and Liang, S.: Atmospheric impacts on climatic variability of surface incident solar radiation, Atmos. Chem. Phys., 12, 9581-9592, doi:10.5194/acp12-9581-2012, 2012.

Weatherhead, E., Reinsel, G., Tiao, G., Ment, X., Choi, D., Cheang, W., Keller, T., DeLuisi, J., Wuebbles, D., Kerr, J., Miller, A., Oltmans, S., and Frederick, E.: Factors affecting the detection of trends: statistical considerations and applications to environmental data, J. Geophys. Res., 103, 17149-17161, 1998.

Wild, M.: How well do IPCC-AR4/CMIP3 climate models simulate global dimming/brightening and twentieth century daytime and nighttime warming?, J. Geophys. Res., 114, D00D11, doi:10.1029/2008JD011372, 2009a.

Wild, M.: Global dimming and brightening: a review, J. Geophys. Res., 114, D00D16, doi:10.1029/2008JD011470, 2009b.

Xia, X.: Spatiotemporal changes in sunshine duration and cloud amount as well as their relationship in China during 1954-2005, J. Geophys. Res., 115, D00K06, doi:10.1029/2009JD012879, 2010a.
Xia, X.: A closer looking at dimming and brightening in China during 1961-2005, Ann. Geophys., 28, 1121-1132, doi:10.5194/angeo-28-1121-2010, 2010b.

Xia, X., Chen, H., Goloub, P., Zhang, W., Chatenet, B., and Wang, P.: A compilation of aerosol optical properties and calculation of direct radiative forcing over an urban region in northern China, J. Geophys. Res., 112, D12203, doi:10.1029/2006JD008119, 2007.

Zhou, L., Dai, A., Dai, Y., Vose, R., Zou, C., Tian, Y., and Chen, H.: Spatial dependence of diurnal temperature range trends on precipitation from 1950 to 2004, Clim. Dynam., 32, 429-440, doi:10.1007/s00382-008-0387-5, 2004.

Zhou, L., Dickinson, R., Dai, A., and Dirmeyer, P.: Detection and attribution of anthropogenic forcing to diurnal temperature range changes from 1950 to 1999: comparing multi-model simulations with observations, Clim. Dynam., 35, 1289-1307, doi:10.1007/s00382-009-0644-2, 2010. 